\title{
Four-Channel Integrated-Optic Wavelength Demultiplexer With Weak Polarization Dependence
}

\author{
Arjen R. Vellekoop and Meint K. Smit
}

\begin{abstract}
A new planar four-channel wavelength demultiplexer with weak polarization dependence based on an optical-phased array, is proposed and demonstrated. An experimental device with dimensions $4.5 \times 3.2 \mathrm{~mm}^{2}$ is designed and fabricated, using conventional (high quality) optical lithography. The demultiplexer operates in the wavelength range 776.5-781.2 nm, with a channel spacing of $1.55 \mathrm{~nm}$. Insertion loss was $0.6 \mathrm{~dB}$ for the central channels and $1.2 \mathrm{~dB}$ for the outer channels for TE polarization, excluding 1.3-dB waveguide propagation loss. Crosstalk values measured 15.4-29.7 dB for the TE and 13.4-22.2 dB for the TM polarization.
\end{abstract}

\section{INTRODUCTION}

$\mathrm{O}$ PTICAL wavelength division multi demultiplexing (WDM) is reckoned to be a useful technique to increase the transmission capacity of a fiber. The key components in a WDM transmission system are the wavelength multiplexers and demultiplexers, for which loss and crosstalk figures, channel spacing, device size, and polarization dependence are important properties.

Various configurations of planar (de)multiplexers have been accomplished by using optical interference filters [1]-[3], wavelength selective coupling [4]-[6], and optical diffraction gratings [7]-[9]. Verbeek et al. [1] reported a weakly polarization-dependent four-channel Mach-Zehnder demultiplexer (device length $33 \mathrm{~mm}$ ) with a channel spacing of $7.7 \mathrm{~nm}, 2.6-\mathrm{dB}$ loss, and 16-dB channel crosstalk. Rottman et al. [4] realized a polarization-independent dual-channel (de)multiplexer with 30-nm channel spacing, 3-dB loss, and 26-40-dB crosstalk, based on two-mode interference (TMI) and with a device length of $12 \mathrm{~mm}$. Imoto et al. [5] proposed and demonstrated a dualchannel directional coupler (de)multiplexer with 100 -nm channel spacing, 5-dB loss, and 20-dB crosstalk. The configuration was designed for a specific polarization and had a length of 10 mm. Suhara et al. [7] demonstrated a five-channel demultiplexer for multimode systems with 30-nm channel spacing using a chirped grating. The hybrid component, with dimensions 20 $\times 13 \mathrm{~mm}^{2}$, showed $9.5-\mathrm{dB}$ loss and $21.5-\mathrm{dB}$ crosstalk. Nonplanar grating components using GRIN lenses [10], [11] achieve better performance. Such devices, however, need to be assembled with great precision and are therefore less attractive.

In this paper a new four-channel wavelength demultiplexer based on an optical phased array is reported. The demultiplexer is weakly polarization dependent and has insertion loss and

Manuscript received September 11, 1989; revised October 2, 1990.

The authors are with the Delft University of Technology, Department of Electrical Engineering Laboratory of Telecommunication and Remote Sensing Technology, 2600GA Delft, The Netherlands. crosstalk figures comparable to the best results reported so far, but with a considerably smaller device size.

\section{BASIC PRINCIPLE}

Recently a planar optical phased array, consisting of a number of concentrically bent waveguides, with both focusing and dispersive properties was reported [12]. The component had considerable loss, however, evidenced by the occurrence of multiple foci in the focal plane, which is characteristic for phased arrays. The power coupled to higher order beams can be reduced by spacing the individual elements more closely. This can be achieved by providing the array of fan-in and fan-out coupling sections at both ends, as shown in Fig. 1. These coupling sections gradually adapt the in- and outcoming beams to the set of guided modes in the array. To obtain a smooth connection between the concentric section and the coupling section, an adapter section is required.

The phase transfer of the complete phased array (including coupling and adapter sections), can be controlled by choosing the radii $R_{i}$ of the concentric section such, that the total length of each channel equals an integer number of wavelengths. This choice of the phase transfer will transform the divergent incoming beam into a convergent outcoming one with the same angular field distribution, so that the source field at the transmitter side will be reproduced in the focal plane at the receiver side. If the array is designed such that the length of the array channels increases linearly with their rank number, then a small variation of the propagation constant $\beta$ will result in a linear variation of the phase transfer which will tilt the outcoming beam and thus lead to a lateral shift of the focal position.

Since the propagation constant $\beta$ in a planar waveguide depends on the polarization as well as the wavelength, the phased array can in principle operate as a polarization splitter as well as a wavelength (de)multiplexer.

The periodic properties of the array function of the phased array have been used to realize a four-channel wavelength demultiplexer with weak polarization dependence.

\section{Theoretical Properties of the Demultiplexer}

The most important physical properties of the demultiplexer are the dispersive lateral focal displacement $d(\Delta \beta)$, the aberration $a(\Delta \beta)$ (shown in the enlargement of the focal plane in Fig. 1), and the insertion loss $L_{\max }$ of the outer channels, which depend on the geometrical parameters of the array as derived in [13]

$$
d(\Delta \beta)=f \cdot \theta(\Delta \beta)=\frac{\Delta \beta}{\beta} \cdot \Psi \cdot\left(f+t+\frac{S_{0}}{2}\right)
$$




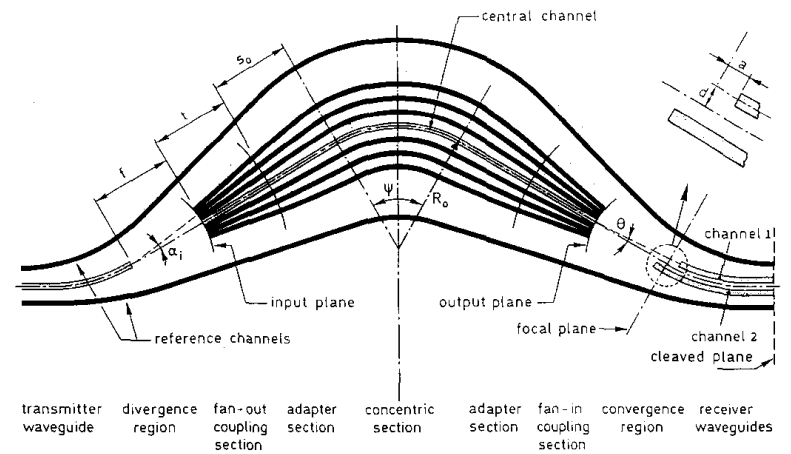

Fig. 1. Demultiplexer geometry. Only two reference channels and two receiver channels are drawn.

$$
\begin{gathered}
a(\Delta \beta)=2 \cdot \frac{\Delta \beta}{\beta} \cdot\left(f+t+\frac{S_{0}}{3}\right) \\
\theta_{\max }=\theta_{0} \cdot \sqrt{\left(\frac{L_{\max }}{20 \cdot \log _{10}(\mathrm{e})}\right)}
\end{gathered}
$$

in which $\Delta \beta$ is the deviation of the propagation constant $\beta$ from the central design value $\beta_{0}, \Psi$ is the concentric-section sector angle, $f$ is the focal length, $t$ is the fan-in and fan-out section length, $s_{0}$ is the adapter central-channel length, $\theta_{0}$ is the beam divergence half angle (at the $1 / \mathrm{e}^{2}$ point) of the single-waveguide far field, described by $\theta_{0}=\lambda / \pi \cdot w_{0}$ with $\lambda$ the wavelength in the film and $w_{0}$ the $1 / \mathrm{e}^{2}$ modal half width, and $\theta_{\max }$ is the maximum dispersion angle (the angular deviation relative to the optical axis as shown in Fig. 1) for which $L \leq L_{\max }$.

Since the propagation constant $\beta$ is dependent on the polarization state of the incoming signal, the phase transfer of the phased array is polarization dependent. If the demultiplexer is designed such that the focal point coincides with the optical axis for the TE polarization at the central wavelength $\lambda_{0}$, then, according to (1), the outcoming field for the TM polarization is tilted by an angle

$$
\Delta \Theta_{p}=\theta_{T E}-\Theta_{T M}=\frac{\Delta \beta_{p}}{\beta f} \cdot \Psi \cdot\left(f+t+\frac{s_{0}}{2}\right)
$$

in which $\theta_{\mathrm{TM}}$ and $\theta_{\mathrm{TE}}$ represent the phase-front angles of the output field for the two polarizations and $\Delta \beta_{p}=\beta_{\mathrm{TE}}-\beta_{\mathrm{TM}}$.

Due to the periodic character of the array function, the phase transfer will be periodic in $\beta$. If the dispersion angle $\Delta \theta_{p}$ equals the period of the array function, the phase transfer of the demultiplexer will be essentially polarization independent: The period $\theta$ of the array function being described by $\sin ^{-1}(\lambda / w)$ $\simeq \lambda / w$, with $\lambda$ the wavelength in the film and $w$ the wavelength width, polarization independence occurs if

$$
\begin{aligned}
\Delta \Theta_{p}=m \cdot \frac{\lambda}{w} & =\frac{2 \pi}{\beta w} \cdot m \\
m & =\cdots-2,-1,0,1,2, \cdots
\end{aligned}
$$

in which $m$ is an arbitrary integer number.

\section{Demultiplexer Design}

A single-mode AlGaAs laser, which is thermally tunable between $775-785 \mathrm{~nm}$, was applied to measure the device, thus fixing the design central wavelength at $\lambda_{0}=780 \mathrm{~nm}$. Designs at longer wavelengths may be realized equally well.

The same (bimodal) waveguide structure (lateral waveguide width $w=2 \mu \mathrm{m}$, optical contrast $\Delta n \simeq 0.02$ ), which was succesfully applied in a polarization splitter [13], was chosen for the demultiplexer. Due to the slightly longer wavelength ( $780 \mathrm{~nm}$ instead of $633 \mathrm{~nm}$ ) the minimal bending radius is increased to $1 \mathrm{~mm}$. The central propagation constant $\beta_{0}(12.46$ $\left.\mu \mathrm{m}^{-1}\right)$, the $1 / \mathrm{e}^{2}$ modal half-width $w_{0}(1.34 \mu \mathrm{m})$ and the difference in propagation constant $\Delta \beta_{p}\left(0.17 \mu \mathrm{m}^{-1}\right)$ of the two polarizations $\beta_{\mathrm{TE}}$ and $\beta_{\mathrm{TM}}$ are fixed with the choice of the waveguide geometry.

As described in Section III, the demultiplexer can be made polarization independent by choosing the parameters such that the angle $\Delta \theta_{p}$ and the array-function period coincide. It then follows from (4) and (5) that

$$
f=\frac{d}{\left(\frac{2 \pi}{\beta w}\right)} \cdot \frac{\Delta \beta_{p}}{\Delta \beta} .
$$

With a lateral receiver-waveguide spacing $d=5 \mu \mathrm{m}$ and a relative-channel spacing $\Delta \beta / \beta_{0}=0.22 \%$, the focal length becomes $123 \mu \mathrm{m}$.

Channel crosstalk considerations are analogous to the polarization splitter design described in [13]. A receiver-waveguide spacing of $5 \mu \mathrm{m}$ and a number $N_{p}=31$ array waveguide should be sufficient for a channel isolation better than $-45 \mathrm{~dB}$. The design parameters and corresponding theoretical properties are summarized in Table I.

It is shown in [13] that if the fan-in and fan-out section length $t$ is chosen at least equal to the focal length, the mutual coupling between adjacent waveguides will become negligible. However, with a value $t=f=123 \mu \mathrm{m}$, the concentric section sector angle $\Psi$, as determined from (1), will be greater than $180^{\circ}$, which can lead to waveguide crossings. For $\Psi$ to become $180^{\circ}$, $t$ has to be chosen $460 \mu \mathrm{m}$.

The first priority in the design considerations was to demonstrate that the phased array could perform a wavelength-demultiplexing function. The demultiplexer was therefore designed for TE polarization. The polarization-independence was achieved by adjusting the focal length according to (6). Consequently, the aberration correction of the receiver channels, as is pointed out in Fig. 1 and in (2), only applies to the TE polarization. The aberration for the TM field is considerable. By substituting $\Delta \beta=\Delta \beta_{p}=0.17 \mu \mathrm{m}^{-1}$ in (2), the aberration $a_{T M}$ becomes $18 \mu \mathrm{m}$. In comparison with a focal depth of $f_{d}=\pi \cdot w_{0} / \lambda=5.4 \mu \mathrm{m}$, being the distance for which the beam waist is less than $w_{0} \cdot \sqrt{2}$ (Gaussian approximation), it can be expected that the demultiplexer properties for TM polarization will be worse than for TE polarization. To average the demultiplexer performances for both polarizations, the aberration correction should be averaged to $\frac{1}{2} \cdot\left(a_{\mathrm{TE}}+a_{\mathrm{TM}}\right)$, so that the receiver waveguides are placed such that the focal mismatch is equal for both polarizations. This correction is not implemented for the present design. An even better solution would be to obtain an aberration-free design. Research on the feasability of such a design is currently being performed.

The theoretical performance of this design can be computed by following the transmitted signal through the demultiplexer as described in [13]. The field at the input plane of the array is calculated as the two-dimensional diffraction field of the fundamental-mode profile at the end of the transmitter waveguide. 


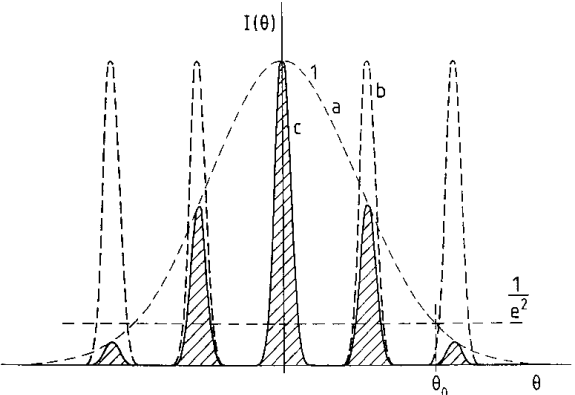

Fig. 2. Field-intensity distribution $I$ as a function of the dispersion angle $\Theta$. The field intensity (pattern $c$ ) is formed by the product of the angular radiation pattern of the singular waveguides (pattern a), and the array function of the phased array (pattern b).

TABLE I

Design Parameters and Calculated Properties of the Four-Channel Wavelength DemultipleXer

\section{wavelength}

central propagation constant (TE)

propagation constant channel spacing

difference $\left(\beta_{\mathrm{TE}}-\beta_{\mathrm{TM}}\right)$

$1 / \mathrm{e}^{2}$ modal half width

$1 / \mathrm{e}^{2}$ beam divergence half angle

focal length

central channel radius

fan-in fan-out section length

transceiver maximum angle

number of waveguides

adapter central length

concentric-section sector angle

dispersion angle channels 1,4

dispersion angle channels 2, 3

displacement channel 1,4

displacement channel 2, 3

aberration channel 1,4

aberration channel 2,3

insertion loss channel 1,4

insertion loss channel 2,3

channel crosstalk

$$
\begin{aligned}
& \lambda_{0}=780 \mathrm{~nm} \\
& \beta_{0}=12.46 \mu \mathrm{m}^{-1} \\
& \Delta \beta=0.0274 \mu \mathrm{m}^{-1} \\
& \Delta \beta_{p}=0.17 \mu \mathrm{m}^{-1} \\
& w_{0}=1.34 \mu \mathrm{m} \\
& \theta_{0}=6.86^{\circ} \\
& f=123 \mu \mathrm{m} \\
& R_{0}=1200 \mu \mathrm{m} \\
& t=460 \mu \mathrm{m} \\
& \alpha_{\max }=14.0^{\circ} \\
& N_{p}=31 \\
& s_{0}=300 \mu \mathrm{m} \\
& \Psi=177.8^{\circ} \\
& \Theta_{1,4}= \pm 3.5^{\circ} \\
& \Theta_{2,3}= \pm 1.16^{\circ} \\
& d_{1,4}= \pm 7.5 \mu \mathrm{m} \\
& d_{2,3}= \pm 2.5 \mu \mathrm{m} \\
& a_{1,4}= \pm 4.5 \mu \mathrm{m} \\
& a_{2,3}= \pm 1.5 \mu \mathrm{m} \\
& L_{1,4}=2.26 \mathrm{~dB} \\
& L_{2,3}=0.25 \mathrm{~dB} \\
& <-45 \mathrm{~dB}
\end{aligned}
$$

Next, coupling of this field into the individual waveguides is computed. The propagation through the array is described by $\exp \left(-j \beta l_{i}\right)$, where $l_{i}$ is the total channel length. At the output plane the field is reconstructed as the sum field of the individual channels from which the two-dimensional diffracted field in the focal plane is calculated (the error made in neglecting coupling effects is cancelled because the same error occurs at the input side). Aberration and truncation effects are inherently accounted for in this simulation.

Fig. 2 depicts the focal field-intensity distribution $I$ as a function of the dispersion angle $\Theta$ (pattern $c$ ), formed by the product of the angular radiation pattern of the singular waveguides (pattern a), and the array function (pattern b). As can be seen from the picture the field intensity decreases with increasing distance of the receiver to the optical axis. This insertion loss is described by (3).

The power transfer is determined by overlapping the field in the focal plane with the modal fields of the receiver waveguides. Results are shown in Fig. 3 as a function of wavelength. The simulation predicts an insertion loss below $2.5 \mathrm{~dB}$ and a crosstalk of $-40 \mathrm{~dB}$, which is $5 \mathrm{~dB}$ higher than the design value

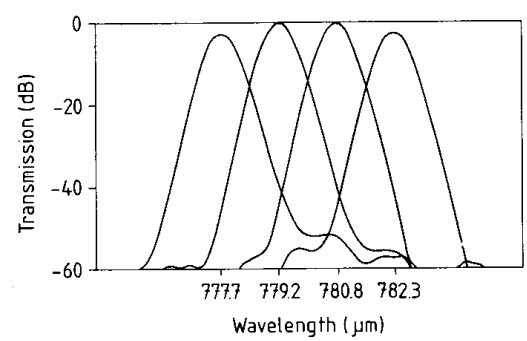

Fig. 3. The numerically simulated power transfer as a function of the wavelength $\lambda$.

due to inclusion of aberration and trunaction effects (as a result of the finite aperture width).

As the demultiplexer is designed for a specific wavelength, deviations from the design wavelength (due to production tolerances or thermal effects) will decrease the demultiplexer performance. Variations within $\pm 10 \%$ of the relative channel spacing $\Delta \beta / \beta_{0}$ have a negligible effect. With the above values, the acceptable relative propagation constant fluctuation lies within $\pm 10 \%$ of $1.7 \mathrm{~nm} / 780 \mathrm{~nm}$, i.e., $\pm 2 \cdot 10^{-4}$, corresponding to a maximum wavelength fluctuation $\Delta \lambda \leq \pm 0.17 \mathrm{~nm}$.

\section{FABRICATION}

The experimental demultiplexer was fabricated in an RF sputter deposited $0.25-\mu \mathrm{m} \mathrm{Al}_{2} \mathrm{O}_{3}$-layer $(n \simeq 1.69$ ) waveguide structure on a thermally oxidized silicon substrate, as described in [14], [15]. The lateral waveguide structure in this layer is produced by atom-beam milling a 40 -nm step and covering the structure with a sputtered $0.6-\mu \mathrm{m} \mathrm{SiO}_{2}$ layer $(n \simeq 1.46)$, so that an embedded ridge guide structure is formed.

The $2-\mu \mathrm{m}$ waveguide pattern is obtained by projecting an optical pattern generator (ASET COMBO 250) created chromium mask onto an image reversal resist film (Hoechst $5214 \mathrm{E}$ ) with a $4 x$ Canon reduction camera (FPA 141).

A photograph of the chromium mask is shown in Fig. 4. The two bent waveguides at the upper side and the two straight waveguides at the lower side of the phased array, are used as reference channels. By comparison of the simultaneously excitated reference channels with the receiver channels, loss and crosstalk values can be determined. The array contains 31 waveguides. Device size is $4.5 \times 3.2 \mathrm{~mm}^{2}$.

\section{RESULTS}

The experimental device was investigated by coupling light into the device from an AlGaAs semiconductor laser operating around $780 \mathrm{~nm}$ with a prism coupler as described in [16]. Input and reference channels were excited simultaneously with a broad beam. Transmission loss was determined by comparing the output intensity to that of the reference channels. Maximum measurement errors, as determined from comparison of identical (straight) waveguides, are within $\pm 0.5 \mathrm{~dB}$. A thermoelectric Peltier module was used to tune the semiconductor laser wavelength between 775 and $785 \mathrm{~nm}$ with a temperature coefficient $d \lambda / d T \simeq 0.25 \mathrm{~nm} / \mathrm{K}$. The cleaved endface of the device, indicated in Fig. 1, is projected onto a CCD video camera with a microscope objective. The camera signal is digitized and processed by a computer. Camera observations at different wavelengths are shown in Fig. 5. Fig. 6 shows a horizontal intensity scan over the picture of Fig. 5(b), from which the insertion loss 


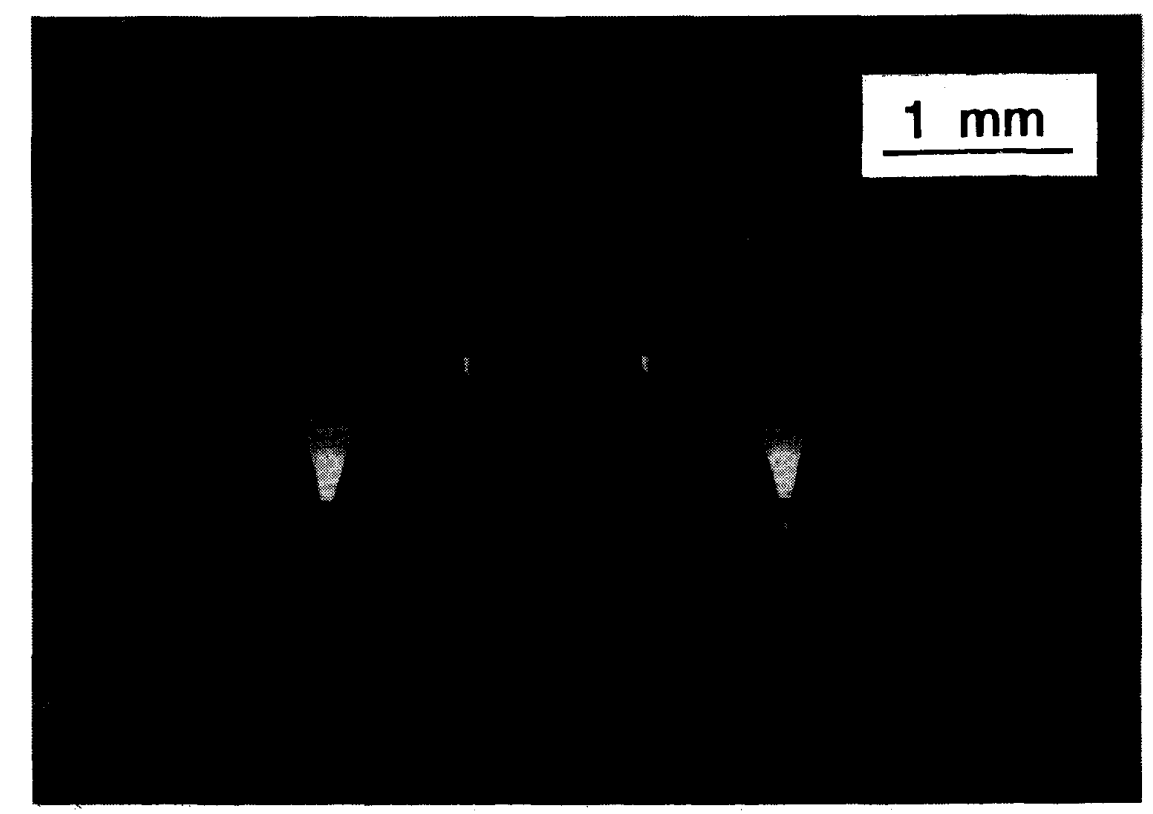

Fig. 4. Mask pattern of the demultiplexer. The upper two bent waveguides and the lower two straight waveguides are reference channels.

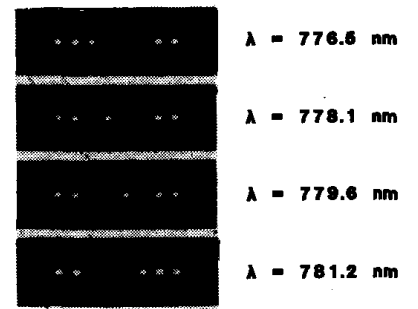

Fig. 5. Camera observations of the channels at the cleaved end face of the device for the four consecutive channel wavelengths.

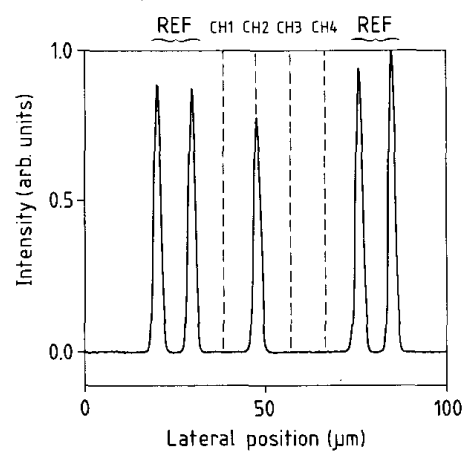

Fig. 6. Intensity scan for $\lambda=778.1 \mathrm{~nm}$ (TE polarization). Estimated insertion loss is $0.6 \mathrm{~dB}$.

can be derived by comparing the intensity from the receiver channel with the bent reference channels (the left two reference channels). Referencing to the bent reference waveguides instead of the straight ones eliminates the effect of waveguide losses which can be seen to amount to $1.3 \mathrm{~dB}$. This loss is fully contributable to the additional $8550-\mu \mathrm{m}$ length of the curved waveguides, and is in good agreement with the $1.5-\mathrm{dB} / \mathrm{cm}$ propagation loss measured in straight waveguides. For the TE polarization the so found insertion loss (excluding waveguide propagation loss) was $0.6 \mathrm{~dB}$ for the central channels and 1.2 $\mathrm{dB}$ for the outer channels, for the TM polarization the insertion loss measured $2.1 \mathrm{~dB}$ for the central and $3.2 \mathrm{~dB}$ for the outer channels.

The TE insertion loss for the outer channel is $1 \mathrm{~dB}$ lower than the theoretically expected value ((3), Table IIa). This may be explained as follows. The limited resolution of the optical lithography will cause filling in of the interwaveguide gap for small gap widths, as can be seen from the photograph (Fig. 4). Due to this filling in, the actual length of the fan-in section will become shorter than the design value. The actual focal length will increase with the same amount.

In the expression for the lateral focal displacement $d(\Delta \beta)$ (1), the dispersion angle $\theta(\Delta \beta)$ will reduce with increasing focal length $f$. The right-hand side of the expression will remain unchanged, however, because the total length $f+t$ will be unchanged. With a reduced dispersion angle $\Theta(\Delta \beta)$ the insertion loss $L$ will reduce according to (3).

The length over which the gaps are filled is experimentally determined to be approximately $70 \mu \mathrm{m}$. With a corrected focal length $f=193 \mu \mathrm{m}$ the actual dispersion angle for the outer channels becomes $2.2^{\circ}$. It then follows from (3) that the maximum insertion loss $L_{\max }=0.9 \mathrm{~dB}$. The measured insertion loss of $1.2 \mathrm{~dB}$ for the outer channels appears to be in good agreement with this corrected value.

Channel isolation is determined by comparing the intensity of the receiver channels, after removing a $20-\mathrm{dB}$ optical attenuator, to that of the reference channels, and measured 15.4$29.7 \mathrm{~dB}$ for the TE polarization and $13.4-22.2 \mathrm{~dB}$ for the TM polarization.

Table II lists the values measured for the demultiplexer. The laser wavelength was measured by coupling part of the input beam to a monochromator with \pm 0.1 -nm resolution. 
TABLE II

Measurement Results of the Demultiplexer for TE- AND TM- POLARIZATION

(Insertion loss and crosstalk values are determined relative to the bent reference waveguides, eliminating waveguide losses.)

\begin{tabular}{cccccc}
\hline & & \multicolumn{5}{c}{ TE transmitted power (dB) } \\
\cline { 3 - 6 } Channel & $\begin{array}{c}\text { Wavelength } \\
(\mathrm{nm})\end{array}$ & 1 & 2 & 3 & 4 \\
\hline 1 & 776.5 & -1.2 & -19.0 & -21.3 & -25.7 \\
2 & 778.1 & -19.4 & -0.6 & -20.0 & -20.6 \\
3 & 779.6 & -17.5 & -16.2 & -0.8 & -15.4 \\
4 & 781.2 & -29.7 & -21.7 & -16.0 & -1.2 \\
\hline
\end{tabular}

\begin{tabular}{cccccc}
\hline & & \multicolumn{4}{c}{ TM transmitted power (dB) } \\
\cline { 3 - 6 } Channel & $\begin{array}{c}\text { Wavelength } \\
(\mathrm{nm})\end{array}$ & 1 & 2 & 3 & 4 \\
\hline 1 & 776.5 & -2.8 & -17.0 & -21.5 & -22.2 \\
2 & 778.1 & -17.6 & -2.1 & -16.5 & -20.0 \\
3 & 779.6 & -20.4 & -17.4 & -3.2 & -15.9 \\
4 & 781.2 & -18.8 & -19.1 & -13.4 & -3.0 \\
\hline
\end{tabular}

The insertion loss and channel isolation for the TM polarization are below the values for TE polarization, due to the large focal aberration occuring for this polarization.

\section{CONClusions}

A novel four-channel wavelength demultiplexer with weak polarization dependence was proposed and demonstrated. The experimental device, realized using conventional high-quality optical lithography, measured an insertion loss (excluding 1.3- $\mathrm{dB}$ waveguide propagation loss) of $0.6 \mathrm{~dB}$ for the central channels and $1.2 \mathrm{~dB}$ for the outer channels for TE polarization, and 2.1 and $3.2 \mathrm{~dB}$, respectively, for TM polarization. Channel isolation was 15.4-29.7 dB for TE and 13.4-22.2 dB for TM polarization, relative to the reference channels. Channel spacing was $1.55 \mathrm{~nm}$. These properties are comparable to the best results reported so far, but realized with a considerably smaller device size.

\section{ACKNOWLEDGMENT}

The authors wish to thank J. W. M. van Uffelen and A. H. de Vreede for fabricating the devices, and Prof. G. A. Acket and Prof. B. H. Verbeek for comments and helpful suggestions.

\section{REFERENCES}

[1] B. H. Verbeek et al. " "Integrated four-channel Mach-Zehnder multi/demultiplexer fabricated with phosphorous doped $\mathrm{SiO}_{2}$ waveguides on Si," J. Lightwave Technol., vol. 6, no. 6, pp. 1011-1015, June 1988.

[2] K. Oda, N. Takato, H. Toba, and K. Nosu, "A wide-band guided-wave periodic multi/demultiplexer with a ring resonator for optical FDM transmission system," J. Lightwave Technol., vol. 6 , no. 6, pp. 1016-1023, June 1988 .

[3] H. Toba, K. Oda, N. Takato, and K. Nosu, "5 GHz-spaced, eight-channel, guided-wave tunable multi-demultiplexer for optical FDM transmission system," Electron. Lett., vol. 23, no. 15 , pp. 788-789, July 1988.

[4] F. Rottmann, A. Neyer, W. Mevenkamp, and E. Voges, "Integrated-optic wavelength multiplexers on lithium niobate based on two-mode interference," J. Lightwave Technol., vol. 6, no. 6, pp. 946-952, June 1988 .

[5] K. Imoto, H. Sano, and M. Miyazaki, "Guided-wave multi/demultiplexers with high stopband rejection," Appl. Opt., vol. 26, no. 19, pp. 4214-4219, Oct. 1987.

[6] R. C. Alferness and R. V. Schmidt, "Tunable optical waveguide directional filter,' Appt. Phys. Lett., vol. 33, no. 2, pp. 161163 , July 1978.

[7] T. Suhara, H. Nishihara, J. Koyama, J. Viljanen, and M. Leppihalme, "Integrated-optic wavelength multi- and demultiplexers using a chirped grating and an ion-exchanged waveguide," in Proc. ECIO. (London, England), Sept. 1981, pp. 12-14.

[8] R. C. Alferness et al., "Narrowband GaInAsP/InP waveguide gratin-folded directional coupler multipexer/demultiplexer,', Electron Lett., vol. 24, no. 3, pp. 150-151, Feb. 1988.

[9] Y. Kokubun, T. Baba, H. Watanabe, and K. Iga, "Monolithic integration of ARROW-type demultiplexer and photodetector," in Proc. ECOC. (Brighton, England), Sept. 1988, pp. 231-234.

[10] J. Lipson et al., "A six-channel wavelength multiplexer and demultiplexer for single mode systems," J. Lightwave Technol., vol. 3 , no. 5 , pp. 1159-1162, Oct. 1985 .

[11] M. Seki et al., "20-channel micro-optic grating demultiplexer for 1.1-1.6 $\mu \mathrm{m}$ band using a small focussing parameter gradedindex rod lens," Electron. Lett., vol. 18, no. 6, pp. 257-258, Mar. 1982.

[12] M. K. Smit, "A new focussing and dispersive planar component based on an optical phased array," Electron. Lett., vol. 24, no. 7, pp. 385-386, Mar. 1988.

[13] A. R. Vellekoop and M. K. Smit, "A small size polarization splitter based on a planar optical phased array," J. Lightwave Technol., vol. 8, no. 1, pp. 118-124, Jan. 1990.

[14] M. K. Smit, C. J. van der Laan, and G. A. Acket, "A1203-films for integrated optics," Thin Solid Films, vol. 138, pp. 171-181, 1986.

[15] M. K. Smit, “'Sputtered planar optical waveguides,' Sensors and Actuators, Procs. S\&A Symposium of the Twente University of Technol. (Enschede, The Netherlands), Oct. 30-31, 1986, pp. 45-55.

[16] W. A. Pasmooij, M. K. Smit, and P. A. Mandersloot, "Selective and stable excitation of planar optical waveguide modes with the prism coupler," J. Lightwave Technol. vol. 7, no. 1, pp. 175180, Jan. 1989.

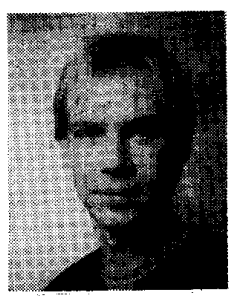

Arjen R. Vellekoop was born in Noordwijkerhout, the Netherlands, on April 11, 1963. He started his study in electrical engineering at the Delft University of Technology, Delft, The Netherlands, in 1981. Since 1986 simultaneously he has studied aerospace engineering. He commenced his master's thesis research in 1987 concentrating on the realization of a polarisation splitter and a wavelength demultiplexer based on an planar optical-phased array.

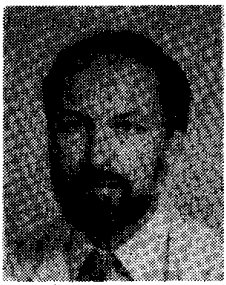

Meint K. Smit was born in Vlissingen, the Netherlands, on January 5, 1951. He graduated in 1974 in electrical engineering at the Delft University of Technology, Delft, the Netherlands.

From 1974 to 1981 he worked in the field of radar remote sensing, firstly as a research assistant to the NIWARS and since 1976 as a staff member of the Delft University. Since 1981 he has worked in the field of integrated optics with an emphasis on passive optical components on silicon substrates for use in optical communication. Since 1986 he has also been engaged in research on the realization of integrated optical circuits in InGaAsP/InP. 